\title{
Total and state-selective electron capture cross sections for $\mathbf{C}^{3+}+\mathbf{H}$ collisions
}

\author{
H C Tseng † and C D Lin† \\ $\dagger$ Department of Physics, Chung Yuan Christian University, Chung Li 32023, Taiwan \\ ‡Department of Physics, Kansas State University, Manhattan, KS 66506, USA
}

Received 21 July 1999

\begin{abstract}
Electron capture cross sections in collisions of $\mathrm{C}^{3+}$ ions with atomic hydrogen have been studied using the close-coupling two-centre atomic orbital (AO) expansion method by treating the collision system in a quasi-two-electron model. Total electron capture cross sections to the dominant individual singlet and triplet excited states are calculated over the energy range 0.1 $50 \mathrm{keV} \mathrm{amu}{ }^{-1}$. The results are compared with existing experimental data and with theoretical calculations based on the molecular orbital (MO) expansion method. It is found that our AO results are in general agreement with the MO results of Errea et al (1991 J. Phys. B: At. Mol. Opt. Phys. 24 4061) and with experiments but that discrepancies still exist in the details.
\end{abstract}

\section{Introduction}

Apart from its fundamental importance, current interest in the $\mathrm{C}^{3+}-\mathrm{H}$ charge changing collision system stems from its possible application in plasma diagnostics and modelling and astrophysics [1]. Several experimental and theoretical studies have been carried out in the last two decades over a broad range of collision energies for this system. The total electron transfer cross section has been measured by Cirić et al [2] at collision energies of 0.92, 1.39 and $1.85 \mathrm{keV} \mathrm{amu}^{-1}$, by Gardner et al [3] at $2.0 \mathrm{keV} \mathrm{amu}^{-1}$, and by Crandall et al [4] at $2.8 \mathrm{keV} \mathrm{amu} u^{-1}$. More extensive measurements have been performed by Phaneuf et al [5] in the 5-112 $\mathrm{keV} \mathrm{amu}^{-1}$ region, by Phaneuf et al [6] in the low-energy range of $10-110 \mathrm{eV} \mathrm{amu}^{-1}$ and more recently by Havener et al [7] in the $0.3-3000 \mathrm{eV} \mathrm{amu}^{-1}$ range using the merged-beam technique. State-selective cross sections have been carried out by both translational energy spectroscopy in the $50-1500 \mathrm{eV} \mathrm{amu}^{-1}$ range [8] and by photon-emission spectroscopy in the 0.7-4.6 $\mathrm{keV} \mathrm{amu}^{-1}$ range [2].

Theoretically, an early calculation of Bienstock et al [9] was based on the full quantal quasimolecular orbital description of the collision but without the inclusion of electron translational motion. They considered cross sections from strong adiabatic coupling to triplet product states $\mathrm{C}^{2+}\left(1 \mathrm{~s}^{2} 2 \mathrm{~s} 3 \mathrm{~s}\right)^{3} \mathrm{~S}, \mathrm{C}^{2+}\left(1 \mathrm{~s}^{2} 2 \mathrm{~s} 3 \mathrm{p}\right)^{3} \mathrm{P}$ and to singlet product states $\mathrm{C}^{2+}\left(1 \mathrm{~s}^{2} 2 \mathrm{p}^{2}\right)^{1} \mathrm{~S}$, $\mathrm{C}^{2+}\left(1 \mathrm{~s}^{2} 2 \mathrm{p}^{2}\right)^{1} \mathrm{D}$. Later, Opradolce et al [10] also performed a molecular model calculation by including the translation effect but their results are significantly different from the results of Bienstock et al [9]. Both of these calculations include only a limited number of states. In a much more elaborate calculation, Errea et al [11] employed 22 molecular states where each state was modified by a common translation factor. Their results are found to be in better general agreement with most of the existing experimental data. However, the large spread of 
experimental total and partial cross sections makes it difficult to draw conclusions on whether the detailed cross sections for this collision system are fully settled.

In view of the above situation, we have undertaken an independent study of the present collision system within the semiclassical close-coupling method by expanding the timedependent electronic wavefunction in terms of atomic orbitals (AO) at the two nuclear centres [12]. Since transitions to the dominant electron capture channels occur mostly at large impact parameters, the $\mathrm{AO}$ expansion method is expected to be adequate down to relatively low energies. In the present calculation, we model $\mathrm{C}^{3+}-\mathrm{H}$ as a two-electron collision system, thus allowing us to obtain state-selective electron capture cross sections to distinct final singlet and triplet states. We used a basis set of about $40-50$ atomic states and the main emphasis is to compare our results with the 22-state MO calculations of Errea et al [11]. In the energy range of interest here, straight-line trajectories were used. In section 2, we document the parameters used in the present calculation. The results and the comparison with experiments and other theoretical calculations are reported in section 3. A short summary and conclusion is given in section 4.

\section{The theoretical model}

We first model $\mathrm{C}^{3+}-\mathrm{H}$ as a two-electron system consisting initially of an electron with the $\mathrm{C}^{4+}$ core and an electron with the proton. The time-dependent wavefunction for these two electrons is expanded in terms of travelling two-electron atomic eigenstates, consisting of configurations where one electron is on the target and the other on the projectile to describe elastic as well as single excitations of either the target or the projectile or both, and of configurations where both electrons are on the projectile for final states populated by the single-electron capture process. The present calculation is carried out using the general two-electron code which has been used previously to study ion-atom collisions [12-14]. For the $\mathrm{C}^{4+}$ core potential we take the screened hydrogenic potential as proposed by Garvey et al [15] with a slightly adjusted thickness parameter of $1 / \xi=0.201$ au and $\eta=4.58$ in order to fit the initial experimental $\mathrm{C}^{3+}(2 \mathrm{~s})$ binding energy. From the model potential one-electron $\mathrm{C}^{3+}$ states and two-electron $\mathrm{C}^{2+}$ states are calculated, the latter using a standard configuration-interaction approach where the two-electron eigenstates are expanded in terms of antisymmetrized products of wavefunctions of two electrons. The dominant electron capture channels considered are $(2 \mathrm{~s} 3 \mathrm{~s})^{3} \mathrm{~S},(2 \mathrm{~s} 3 \mathrm{p})^{3} \mathrm{P}$, $(2 \mathrm{~s} 3 \mathrm{~d})^{3} \mathrm{D}$ for triplet states and $(2 \mathrm{~s} 3 \mathrm{~s}){ }^{1} \mathrm{~S},\left(2 \mathrm{p}^{2}\right)^{1} \mathrm{~S},(2 \mathrm{~s} 3 \mathrm{p})^{1} \mathrm{P},\left(2 \mathrm{p}^{2}\right)^{1} \mathrm{D},(2 \mathrm{~s} 3 \mathrm{~d}){ }^{1} \mathrm{D}$ for singlet states. We compare in table 1 the calculated energies of these states from the model potential with the experimental values.

In performing the close-coupling calculation the total spin is conserved, thus calculations were carried out separately for spin singlet and spin triplet symmetries. In each calculation, the two-electron states on the projectile consist of those listed above, together with a number of pseudostates generated from the basis functions. The products of one-electron states on each centre consist of the initial state, the single excitation of the target to $2 \mathrm{~s}$ or $2 \mathrm{p}$, and the single excitation of the projectile from $2 \mathrm{~s}$ to $2 \mathrm{p}$. The total number of atomic states included in the close-coupling calculations is 36 for singlet symmetry and 40 for triplet symmetry, but in test calculations the number has been raised to about 50 by including more pseudostates to check the convergence. With the basis functions chosen, the standard procedure for performing close-coupling calculations is used to obtain scattering cross sections to individual final states. We considered straight-line trajectories only in the calculations. We stopped our calculations at $100 \mathrm{eV} \mathrm{amu}^{-1}$ since at lower energies the Coulomb repulsion between $\mathrm{C}^{4+}$ and $\mathrm{H}^{+}$is no longer negligible. Thus the present calculation is a genuine $\mathrm{AO}$ calculation within the basis set and the model potential chosen. 
Table 1. Comparison of energy levels in atomic units for the $\mathrm{C}^{2+}$ ion from the present model potential calculations and the experimental data [18]. Energies are given relative to the $\mathrm{C}^{4+}$ ion.

\begin{tabular}{lll}
\hline Two-electron state & Present work & Experimental value \\
\hline $2 \mathrm{~s}^{2}{ }^{1} \mathrm{~S}^{\mathrm{e}}$ & -4.13 & -4.13 \\
$2 \mathrm{p}^{2}{ }^{1} \mathrm{~S}^{\mathrm{e}}$ & -3.27 & -3.30 \\
$2 \mathrm{~s} 3 \mathrm{~s}^{1(3)} \mathrm{S}^{\mathrm{e}}$ & $-2.96(-3.04)$ & $-3.00(-3.04)$ \\
$2 \mathrm{p} 3 \mathrm{p}{ }^{1(3)} \mathrm{S}^{\mathrm{e}}$ & $-2.55(-2.64)$ & $-2.56(-2.64)$ \\
$2 \mathrm{~s} 2 \mathrm{p}^{1(3)} \mathrm{P}^{\mathrm{e}}$ & $-3.60(-3.88)$ & $-3.67(-3.89)$ \\
$2 \mathrm{~s} 3 \mathrm{p}^{1(3)} \mathrm{P}^{\mathrm{e}}$ & $-2.94(-2.94)$ & $-2.95(-2.95)$ \\
$2 \mathrm{p} 3 \mathrm{~s}^{1(3)} \mathrm{P}^{\mathrm{e}}$ & $-2.65(-2.72)$ & $-2.72(-2.73)$ \\
$2 \mathrm{p} 3 \mathrm{~d}^{1(3)} \mathrm{P}^{\mathrm{e}}$ & $-2.54(-2.56)$ & $-2.55(-2.58)$ \\
$2 \mathrm{p} 3 \mathrm{~d}^{1(3)} \mathrm{P}^{\mathrm{e}}$ & $-2.54(-2.56)$ & $-2.55(-2.58)$ \\
$2 \mathrm{p}^{2} \mathrm{D}^{\mathrm{e}}$ & -3.46 & -3.47 \\
$2 \mathrm{~s} 3 \mathrm{~d}^{1(3)} \mathrm{D}^{\mathrm{e}}$ & $-2.86(-2.89)$ & $-2.87(-2.90)$ \\
$2 \mathrm{~s} 4 \mathrm{~d}^{3} \mathrm{D}^{\mathrm{e}}$ & -2.66 & -2.67 \\
$2 \mathrm{p} 3 \mathrm{p}^{1(3)} \mathrm{D}^{\mathrm{e}}$ & $-2.61(-2.64)$ & $-2.61(-2.66)$ \\
\hline
\end{tabular}

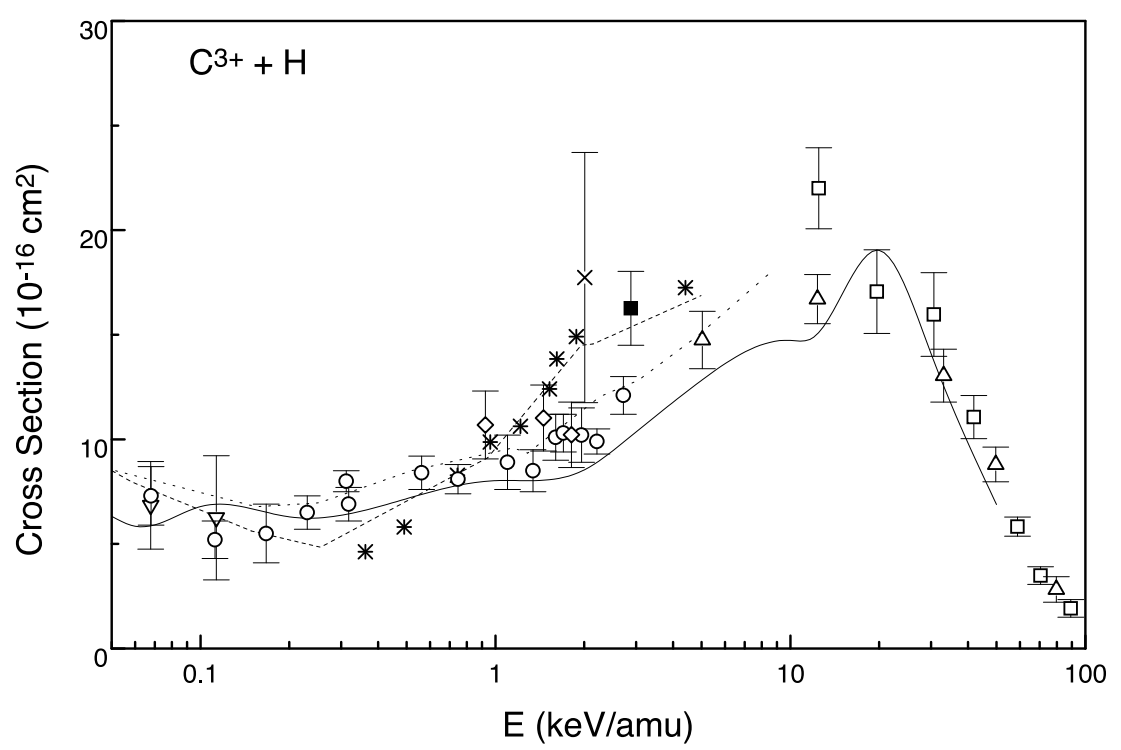

Figure 1. Present total electron capture cross section results for $\mathrm{C}^{3+}+\mathrm{H}$ compared with other theoretical and experimental data. Theoretical results: solid curve, present work; dashed curve, Bienstock et al [9]; dotted curve, Errea et al [11]. Experimental results: open circles, Havener et al [7]; open up triangle, Phaneuf et al [5]; open down triangle, Phaneuf et al [6]; open squares, Goffe et al [17]; solid squares, Crandall et al [4]; stars, Yousif and Geddes [16]; crosses, Gardner et al [3]; open diamond, Ćirić et al [2].

\section{Results and discussion}

In figure 1 we compare the available experimental and theoretical total electron capture cross sections in the energy range of $50 \mathrm{eV} \mathrm{amu}^{-1}$ to $100 \mathrm{keV} \mathrm{amu}^{-1}$. Within the error bars of the experiments there is an overall global agreement. Our results have good agreement with the experimental data from $100 \mathrm{eV} \mathrm{amu}^{-1}$ to $100 \mathrm{keV} \mathrm{amu}^{-1}$, except that we tend to underestimate the experimental data by about $20 \%$ in the energy region of $1-5 \mathrm{keV} \mathrm{amu}^{-1}$. 
In this energy region the calculations of Errea $e t$ al have the better overall agreement with experiments. Within $400-4000 \mathrm{eV} \mathrm{amu}^{-1}$ the data of Yousif and Geddes [16] appear to deviate from other experiments but they are in good agreement with the calculation of Bienstock et al [9]. However, the latter calculations were carried out with a small basis set and thus we believe that both of these two results should be taken less seriously. If we also remove the two singular measured points, as indicated by the cross and by the solid square, and the lowest energy point indicated by the open square, then we can conclude that the total charge transfer cross section for the present system is known to within about $20 \%$. Between the two extensive calculations, the results of Errea et al are consistently 10-20\% higher than the present results.

For the present system there are two measurements of the state-selective electron capture cross sections, one from the translational energy spectroscopy measurements of Wilkie $e t a l$ and the other from the photon-emission spectroscopy measurement of Ćiric et al. We compare the theoretical results with these two measurements for energies between $100-10000 \mathrm{eV} \mathrm{amu}^{-1}$ in figures 2 and 3. We note that in this energy region the dominant electron capture is to the $(2 \mathrm{~s} 3 \mathrm{~s})^{3} \mathrm{~S}$ state. We show the comparison of the results for this state in figure $2(a)$. The calculations of Errea et al agree quite well with the data of Ćirić et al in the energy region where they overlap. Interestingly, the calculations of Bienstock et al are in good agreement with the data of Wilkie et al. However, these two MO calculations do not agree with each other. Our AO results are in better agreement with the MO results of Errea et al in terms of the energy dependence, but we are consistently about $20 \%$ lower than theirs in terms of absolute cross sections.

We next check the weaker channels. In figure $2(b)$ the cross sections for electron capture to the $\left(2 p^{2}\right)^{1} S$ state are shown. Our results are in good agreement with Errea et al for the whole energy range displayed but both calculations are higher than the experimental data of Ćirić et al at higher energies. Similarly for capture to the $\left(2 \mathrm{p}^{2}\right)^{1} \mathrm{D}$ state, as shown in figure $2(c)$, our calculations are closer to the results of Errea $e t a l$ and agree well with the experiments of Wilkie et al, but we are consistently higher than the data of Ćirić et al.

In figures $3(a)-(e)$, we compare the theoretical calculations with the experimental data of Ćiric et al. The overall agreement is satisfactory in view of the fact that these are the weaker channels. Our predicted state-selective cross sections are, in general, quite close to the calculations of Errea et al except for the case of the $(2 \mathrm{~s} 3 \mathrm{~s})^{1} \mathrm{~S}$ state, especially at low energies. This may be due to the fact that we did not achieve a good fit of the experimental energy for this state (see table 1). We comment that in most cases the results of Errea et al appear to be in better agreement with the data of Ćiric et al.

Returning to figure $2(a)$, it is surprising to find that there is a consistent discrepancy between the present calculation and the MO result of Errea et al for electron capture to the dominant $(2 \mathrm{~s} 3 \mathrm{~s})^{3} \mathrm{~S}$ state, especially in the $1-10 \mathrm{keV} \mathrm{amu}{ }^{-1}$ region. It is noted that from the MO picture transition to this state occurs primarily through the avoided crossing near $R=11.7$ au. At this distance the two MO states are essentially atomic in nature and thus the AO expansion method is expected to be as good as the MO method. In figure 4 we show the comparison of the electron capture probabilities calculated by Errea et al and from the present $\mathrm{AO}$ calculation at $2.25 \mathrm{keV} \mathrm{amu}{ }^{-1}$. While the shapes of the electron capture probabilities appear to be quite similar, the MO calculation clearly has larger probabilities at larger impact parameters and at smaller impact parameters. While the discrepancy at smaller impact parameters can be expected because of the nature of the basis functions used in the two calculations, the discrepancy at larger impact parameters is less clear since the basis functions in the two calculations are essentially identical, the only difference is in the form of electron translational factors. We also checked the difference of the model potentials used in the two calculations, and there is no significant distinction between the two. However, it is noted that a 

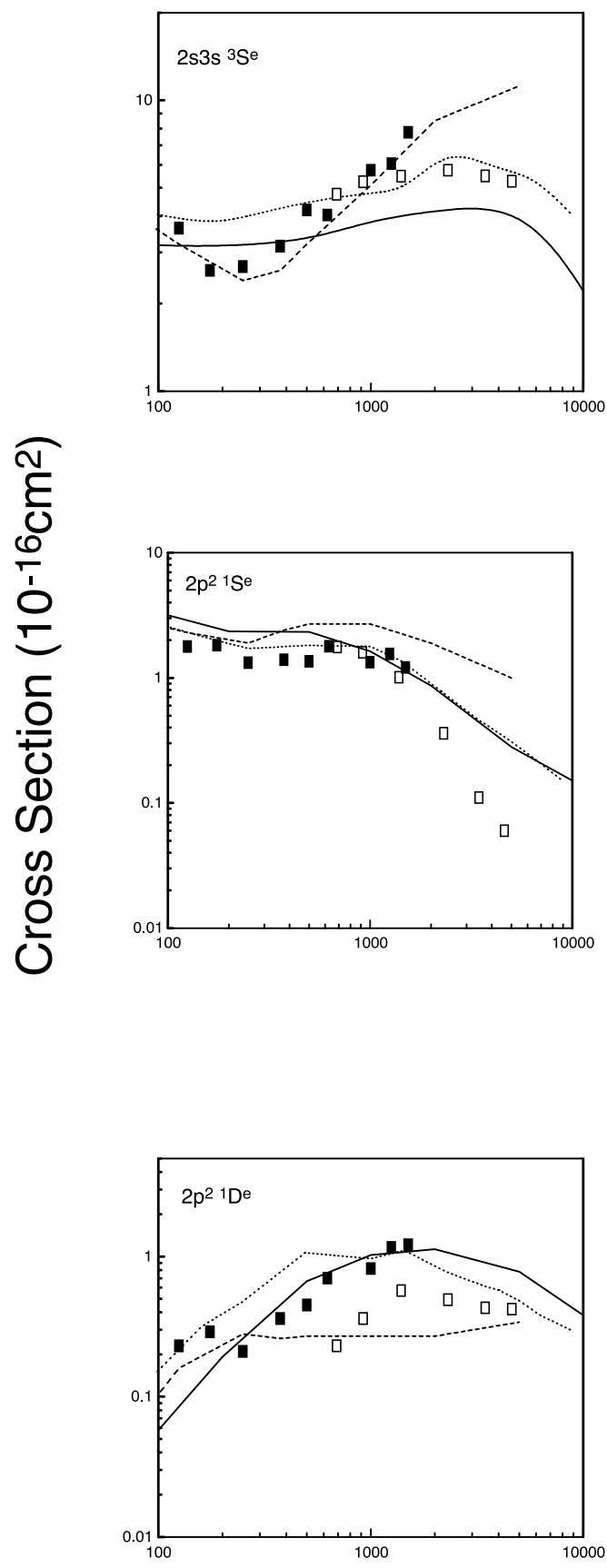

Figure 2. Comparison of theoretical results and experimental data for state-selective electron capture cross sections. Solid curve, present work; dashed curve, Bienstock et al [9]; dotted curve, Errea et al $\mathrm{E}(\mathrm{eV} / \mathrm{amu})$ [11]. Experiments: solid squares, Wilkie et al [8]; open squares, Ćirić et al [2].

non-local Phillips-Kleinmann pseudopotential was used to shift all virtual MO (or core) states to higher energies to avoid possible spurious curve crossing in the calculation of Errea et al. 

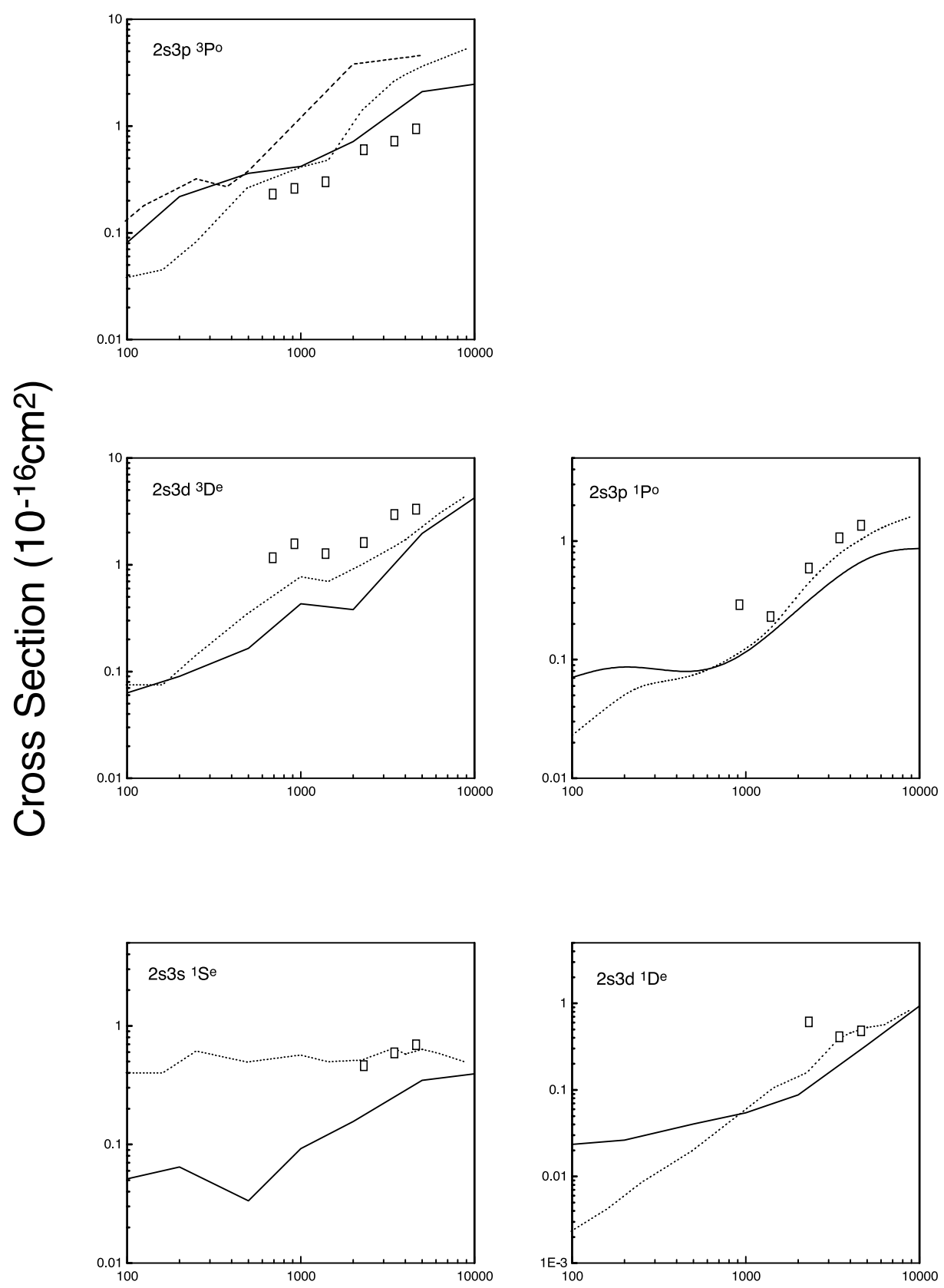

\section{Projectile energy (eV/amu)}

Figure 3. Comparison of theoretical results and experimental data for state-selective electron capture cross sections. Solid curve, present work; dashed curve, Bienstock et al [9]; dotted curve, Errea et al [11]; open squares, experimental data of Ćirić et al [2]. 


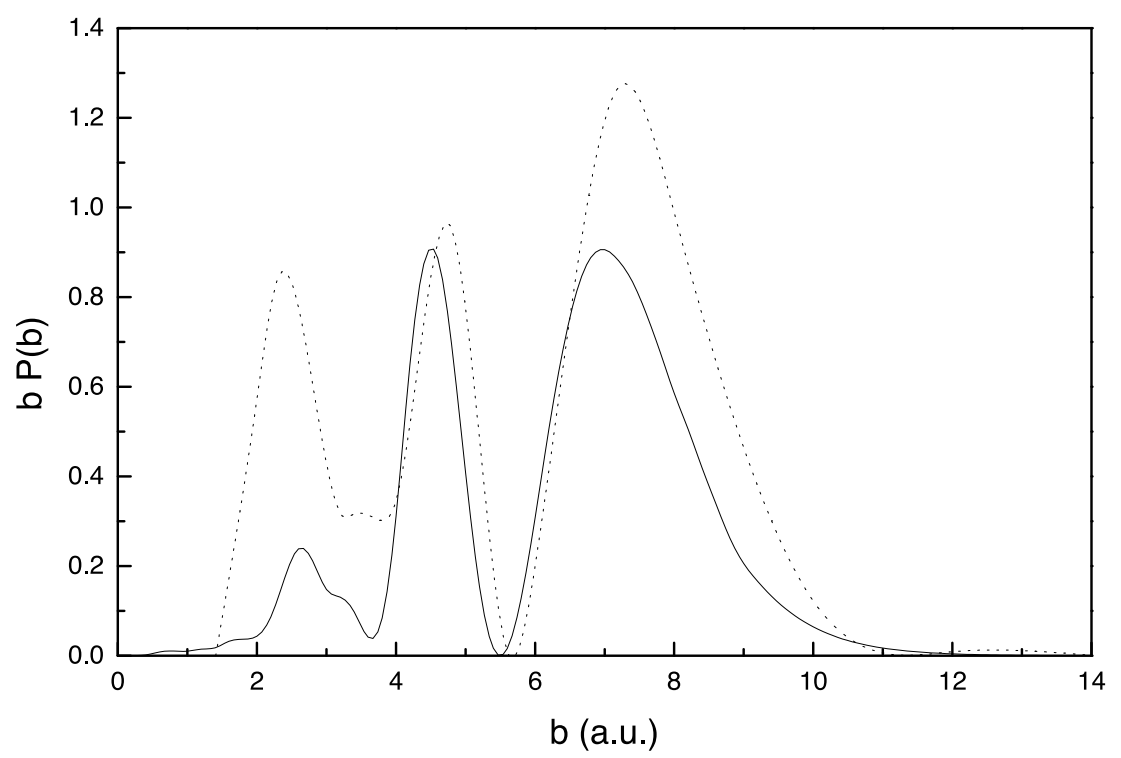

Figure 4. Impact parameter dependence of electron capture probability $P(b)$ to the $(2 \mathrm{~s} 3 \mathrm{~s}){ }^{3} \mathrm{~S}$ state times impact parameter $b$ at $2.25 \mathrm{keV} \mathrm{amu}^{-1}$. Solid curve, present work; dotted curve, Errea et al [11].

\section{Summary and conclusion}

In this paper we report the total and state-selective single-electron capture cross sections for $\mathrm{C}^{3+}$ on $\mathrm{H}$ collisions over the energy range of $100-100000 \mathrm{eV} \mathrm{amu}^{-1}$ using the close-coupling expansion method in terms of two-electron atomic basis functions. In this energy region the trajectory effect is negligible and thus the present calculation is a genuine AO calculation without any corrections. In terms of the total electron capture cross sections, there is a general agreement among the experiments and the more advanced theoretical calculations. Our results are shown to be in best overall agreement with the 22-state MO calculations of Errea et al. For the state-selective electron capture cross sections the agreement between the two calculations is also satisfactory. However, there still exists a 10-20\% discrepancy in the total cross sections which probably can be resolved by a much more dedicated experimental and theoretical effort in the future.

\section{Acknowledgments}

This work is supported in part by the National Science Council, Taiwan (HCT) and the Division of Chemical Science, Office of Basic Energy Sciences, Office of Science, US Department of Energy (CDL).

\section{References}

[1] Gilbody H B 1994 Adv. At. Mol. Phys. 32149

[2] Ćirić D, Brazuk A, Dijkkamp D, de Heer F J and Winter H 1985 J. Phys. B: At. Mol. Phys. 183629

[3] Gardner L D, Bayfield J E, Koch P M, Sellin I A, Pegg D J, Peterso R S and Crandall D H 1980 Phys. Rev. A 211397

[4] Crandall D H, Phaneuf R A and Meyer F W 1979 Phys. Rev. A 19504 
[5] Phaneuf R A, Meyer F W and McKnight R H 1978 Phys. Rev. A 17534

[6] Phaneuf R A, Alvarez I, Meyer F W and Crandall D H 1982 Phys. Rev. A 261892

[7] Havener C C, Muller A, Zeijlmans van Emmichoven P A and Phaneuf R A 1995 Phys. Rev. A 512982

[8] Wilkie F G, Yousif F B, McCullough R W and Gilbody H B 1986 J. Phys. B: At. Mol. Phys. 19239

[9] Bienstock S, Heil T G, Bottcher C and Dalgarno A 1982 Phys. Rev. A 252850

[10] Opradolce L, Benmeuraiem L, McCarroll R and Piacentini R D 1988 J. Phys. B: At. Mol. Opt. Phys. 21503

[11] Errea L F, Herrero B, Mendez L and Riera A 1991 J. Phys. B: At. Mol. Opt. Phys. 244061

[12] Fritsch W and Lin C D 1991 Phys. Rep. 2021

[13] Fritsch W and Lin C D 1988 Phys. Rev. Lett. 46690

[14] Fritsch W and Lin C D 1988 J. Phys. B: At. Mol. Opt. Phys. 192683

[15] Garvey R H, Jackman C H and Green A E S 1975 Phys. Rev. A 121144

[16] Yousif F B and Geddes J (unpublished data cited in [7])

[17] Goffe T V, Shah M B and Gilbody H B 1979 J. Phys. B: At. Mol. Phys. 123763

[18] Moore C 1974 Atomic Energy Levels (NBS Circular No 467) (Washington, DC: US Govt Printing Office) 